Humanitarian mapping as library outreach: A case for community-oriented mapathons

Theresa Marguerite Quill

Indiana University Bloomington

theward@indiana.edu 


\begin{abstract}
Maps and geospatial data are critical in disaster response situations. Accurate and updated maps direct first responders to areas of most need, reroute supply lines according to the post-disaster landscape, and help identify remote communities. Unfortunately, accurate and detailed geospatial data is not readily available for many parts of the world. Crowdsourced mapping programs such as Humanitarian OpenStreetMap (HOT) and Tomnod rely on volunteers to create this essential data, with a focus on the world's most vulnerable places. Groups of volunteers contribute to HOT and Tomnod in events called mapathons. This case study at Indiana University Bloomington's Herman B Wells Library asserts that the library is a natural home for humanitarian mapathons, as participants actively engage with spatial and data literacy concepts as they become spatial data creators. Through library mapathons, participants gain spatial and data literacy skills, engage with a global community, connect with other parts of the world, and are exposed to library resources. Hosting a mapathon requires very few specialized skills or knowledge, and has broad appeal. While Indiana University Bloomington serves about 40,000 students, this case study provides tips and best practices for hosting humanitarian mapathons at libraries of any size.
\end{abstract}

\title{
Keywords
}

Participatory Geographic Information Systems, PPGIS, Volunteered Geographic Information, Humanitarian Mapping, Mapathons, Humanitarian OpenStreetMap, Interdisciplinary library outreach 


\section{Humanitarian mapping as library outreach: A case for community-oriented mapathons}

Participatory Geographic Information Systems (PPGIS) and Volunteered Geographic Information (VGI) are growing trends in the geospatial community. Similar to other crowdsourced projects like Wikipedia, community-created maps have great potential as a means of engaging library users with data and information creation. Volunteer humanitarian mapping provides a connection to places in crisis and promotes geographic literacy and global engagement. This form of digital humanitarianism can be especially powerful for diaspora communities, or anyone wanting to contribute to impactful humanitarian relief in a nonmonetary way.

This paper examines two vehicles for volunteered humanitarian mapping: OpenStreetMap (OSM) and Tomnod, and their use in the library. Humanitarian OpenStreetMap (HOT) mapathons have gained popularity in academic libraries, particularly after hurricanes Harvey, Irma, and Maria devastated areas of the United States and the Caribbean in 2017 (Peet 2017). The case study of Indiana University's Herman B Wells Library, an academic library serving a student population of about 40,000 students, is replicable in libraries of all types, including public libraries, and requires no specialized skills or equipment. The library is a natural home for this type of activity, as mapathons offer a real-world opportunity to engage with spatial and data literacy skills as participants become spatial data creators.

\section{Humanitarian mapping overview}

Maps and geospatial information are critical in disaster relief and prevention. Accurate geospatial data and analysis can help predict where a disaster might occur, inform evacuation 
planning, and essentially, direct search and rescue teams to where they are most needed. A report from the National Research Council concluded:

"In all aspects of emergency management, geospatial data and tools have the potential to contribute to the saving of lives, the limitation of damage, and the reduction in the costs to society of dealing with emergencies. Responders who know where impacts are greatest, where critical assets are stored, or where infrastructure is likely to be damaged are able to act more quickly, especially during the "golden hour" immediately after the event when there is the greatest possibility of saving lives." (NRC 2006, 2)

Prior to the use of PPGIS in disaster management, first responders and aid organizations relied solely on traditional organizational structures for mapping and geospatial information. Governments are often the largest producers of geospatial information and are seen as the most authoritative sources of cartographic information. However, in part due to the level of detail and accuracy in government geospatial information, products from government sources take longer to produce, and can be difficult to access in some parts of the world (Nourbakhsh et al. 2006). In addition, the 2007 National Research Council report details struggles with information sharing across government agencies and issues with use rights for information created by private entities. While governmental mapping agencies and private companies continue to be the standard bearers for accurate geospatial data, PPGIS has emerged as an agile and democratic alternative for geospatial data creation in disaster response.

PPGIS projects like OpenStreetMap rely on thousands of volunteers to update maps and quickly create detailed geospatial information. These projects disrupt the traditional models of cartography and have democratized the process of mapping, giving agency to lay people to literally put themselves on the map (Tulloch 2007; Lin 2011). Given the importance of geospatial information in disaster response, a growing interest in citizen science, and the growth of new mapping technologies like OpenStreetMap that allow lay people to view and map the 
earth in a way that was previously only available to geospatial professionals, it seems natural that PPGIS would have emerged as a tool for humanitarian mapping.

However, accuracy and data quality is a concern with PPGIS. Lacking the authority and structures for data assessment that traditional producers of spatial data rely on, accuracy of PPGIS data depends on a number of factors, including clarity of objectives, quality of base map information, and number of participants (Brown 2012; Elwood, Goodchild, and Sui 2012). In general, the more people contributing and checking data in a particular area, the more accurate the data will be (Elwood, Goodchild, and Sui 2012). The idea with PPGIS and other crowdsourced projects is that a large number of contributors will arrive at a collective truth or accuracy. As Elwood, Goodchild, and Sui point out: all geospatial data is limited, as it is an abstraction and simplification of the world (2012). In the end, users of data must decide for themselves if the dataset is appropriately authoritative and accurate for their needs. For the type of spatial data collected by OSM and HOT (describing locations of roads and buildings), PPGIS data, and in particular OSM, has been found to be at least as accurate as available traditional sources of spatial data (Haklay 2010; Brown 2012; Elwood, Goodchild, and Sui 2012). While skepticism and criticisms of PPGIS should not be dismissed, the utility of PPGIS in timesensitive post disaster situations is undeniable.

\section{Mapping tools overview}

OpenStreetMap (OSM) was created in 2004 as a free, crowdsourced alternative to Ordinance Survey maps in the United Kingdom. Since its inception, OSM has grown to be an open source, detailed, and accurate map of the world. ("OpenStreetMap" n.d.). The map is created by the OSM community, which includes lay person volunteers, Geographic Information 
Systems (GIS) experts, software engineers, and digital humanitarians. Humanitarian OpenStreetMap is a sister organization that grew alongside OSM and focuses on improving OSM for use by relief agencies for disaster response. HOT's first large-scale mission was after the 2010 earthquake in Haiti. After the earthquake struck, it quickly became apparent that not only were there no up to date and detailed maps of Haiti, but that disruption of communication networks meant that in many cases, actors outside of Haiti had more information than people on the ground. HOT volunteers around the world traced satellite imagery and older CIA maps of Haiti to fill in the gaps of knowledge (Mikel 2010). HOT then shared this geospatial data with relief agencies to inform where they were most needed.

Since 2010, HOT has grown to include partners such as Missing Maps and the US Department of State MapGive initiative that help train volunteers and promote the use of Humanitarian OpenStreetMap data. According to MissingMaps.org, over 42,000 volunteers have contributed to the map since 2014. This map data is actively used by organizations such as the Red Cross and Doctors Without Borders ("Missing Maps" n.d.). More recent campaigns include mapping remote areas of Nepal after the 2015 earthquake, and mapping areas affected by Hurricanes Harvey, Maria, and Irma in 2017.

Another map-based humanitarian tool that has potential for library programming is Tomnod. Rather than creating the map itself, Tomnod volunteers search satellite imagery provided by the parent company, DigitalGlobe, and tag areas of interest. Past campaigns have included searching for wreckage of Malaysian Airlines Flight 370, tagging post-hurricane landslides and damaged buildings, and searching for Weddell Seals in Antarctica.

\section{Benefits for mapathon participants}


Both Humanitarian OpenStreetMap and Tomnod are powerful humanitarian tools that are impactful in a library environment. The barrier to entry for both of these tools is low, and appeals to a diverse population of potential mappers (Lin 2011). Participants in humanitarian mapping library events gain a sense of global engagement and are able to connect to places around the world in crisis. In a survey of OpenStreetMap contributors, Lin (2011) found that a key motivation for participation in OSM was an emotional or personal connection to a place. "Mapping, just like blogging, is a personal way of expressing, preserving memories, documenting one's footprints, a way of travelling and a way of remembering events in everyday life" (Lin 2011, 61). This type of action can be especially poignant for diaspora communities when they are away from friends and family in a time of crisis. Mapathon events held at Indiana University's Herman B Wells Library after the 2015 Nepal earthquake and Hurricane Maria in 2017 drew a diverse population, but both events included international students and community members from the affected areas. The mapathons provided a space for people from the affected areas to talk to other community members, to share stories of the places that were destroyed, and to educate others about places that they had an emotional connection with. Beyond the actual contributions to HOT or Tomnod, this type of cross-cultural conversation is one of the major benefits to hosting humanitarian mapathons in the library.

Participants also develop spatial literacy skills as they learn core concepts of mapping and GIS technology. Mapathons that provide basic training for HOT and Tomnod often lead to conversations about the usefulness and authority of maps, and provide a natural segue to discuss how the maps that we use every day are created and what biases they might contain. Discussions of geographic literacy concepts are obviously not required for hosting a mapathon, but can 
naturally occur as participants engage with creating their own spatial data, and are opportunities for librarians to engage participants in deeper data literacy concepts.

\section{Hosting a mapathon: Herman B Wells Library case study}

Hosting a mapathon requires very little. Missing Maps

(http://www.missingmaps.org/host/) provides a comprehensive checklist for HOT mapathon planning. Other excellent training materials can be found at Humanitarian OpenStreetMap (https://www.hotosm.org/get-involved/disaster-mapping) and MapGive

(https://mapgive.state.gov/learn-to-map/). Prior to hosing a mapathon, facilitators should complete the MapGive "learn to map" tutorial and spend enough time practicing in OpenStreetMap that they feel comfortable leading participants through the tutorial and troubleshooting during the session. Depending on prior experience and technology skills, this may take between 1-4 hours. While having a background in GIS will expedite this process, it is definitely not necessary. Anyone comfortable using a computer can learn to host a mapathon. While all of the practicalities of learning to contribute to contribute to HOT and host a mapathon have been well documented by the previously listed organizations and the practical guide at the end of this article, effectiveness of library mapathons depends on tailoring the events to the community.

In the three years that Herman B Wells Library has been hosting humanitarian mapathons, we have gone through many trials and errors, and experimented with the format of hosting and promoting mapathons. Mapathons at Herman B Wells take place in the context of a semester-long series of mapping workshops. These workshops are targeted at graduate students, but open to anyone. Each semester includes a general workshop on humanitarian mapping, 
where participants meet in a computer lab and learn to contribute to OpenStreetMap. These introductory workshops begin with a discussion by the workshop presenter about the importance of maps and geospatial data in crisis situations, the impact of HOT, as well as the everyday uses of OpenStreetMap data in non-humanitarian applications. The discussion is followed by a live tutorial on how to contribute to OSM, with links to video tutorials if participants prefer to learn at their own speed. Most of the hour-long workshop time is used for participants to experiment with making edits to OSM independently. In all mapathons, it is useful to have more than one workshop facilitator who is able to float around the room and individually assist participants. Over the years, we have held Intro to OSM workshops in a variety of locations, but the most successful have been in a collaborative computer lab space where multiple people can work on one computer screen. As OSM is a collaborative project at its core, allowing participants to work together on the map fosters discussion and allows more hesitant participants to engage in a less direct way. The Intro to OSM workshops are advertised as hour-long workshops where participants are expected to attend for the duration. The intention behind hosting regular introductory OSM workshops is to build a community of mappers who are familiar with OSM and the concept of humanitarian mapping, so that when disaster strikes, there is a group in place ready to contribute to a humanitarian mapathon. The library maintains a listserv to send communications about humanitarian mapping events that is advertised at the introductory sessions. These mapathons are open to all. University-affiliated and community members are welcome to attend.

The majority of mapathons at Indiana University are in response to a disaster. The first of these humanitarian mapathons was after the 2015 earthquake in Nepal. Indiana University has a significant Nepali student population, and the mapathon was part of a larger response from the 
University that included a candlelight vigil, poetry readings, fundraiser, and other events. Since then, the Herman B Wells Library has hosted humanitarian mapathons after several other disasters, most recently the 2017 Atlantic hurricanes. The structure of disaster-response mapathons differs from introductory OSM workshops. Most of the time, humanitarian mapathons are scheduled with very little advance notice, and take place in whichever venue is available. Participants are often asked to provide their own laptops for these events, and they are structured as a drop-in activity. We have had success hosting humanitarian mapathons on a large visualization wall, as the wall allows for many people to view the same detailed area. Participants can also plug up to four laptops into the wall and view four screens at once. The visualization wall area is open and visible, and has a more inviting setup than a traditional computer lab. It is not uncommon for someone to walk into a humanitarian mapathon held at the visualization wall because they were walking by and the event looked interesting.

Rather than focusing on Humanitarian OpenStreetMap, the drop-in nature of humanitarian mapathons lends itself to using Tomnod as another mapping option. Tomnod provides satellite imagery that participants analyze to tag areas that appear to have been impacted by an event. The benefit of using Tomnod rather than HOT in these situations is that Tomnod takes less time to learn, and can be more collaborative on the visualization wall. Multiple participants can stand around the visualization wall and scan for areas to tag, often leading to discussions and debates about what the satellite imagery is showing. In most of the humanitarian mapathons at Herman B Wells Library some participants will choose to work with HOT, most often people who have attended the introductory sessions, while drop-in participants tend to prefer Tomnod. 


\section{Practical steps for hosting a mapathon}

\section{Before the event:}

1. Facilitators complete MapGive tutorials (https://mapgive.state.gov/learn-to-map) and practice editing OSM.

2. Recruit a co-facilitator if possible!

3. Book a space. Collaborative spaces tend to work best.

4. Advertise the event widely.

a. Reach out to organizations who may want to partner: diaspora communities, student organizations, area studies librarians, etc.

b. If participants need to bring their own laptops, be sure to make that clear.

c. Provide links to training materials if people want to practice before attending the mapathon.

5. Arrange for snacks if possible.

6. Consider library materials and collections that you might bring to the event. These might include GIS or mapping resources, or materials about the part of the world that you are supporting.

\section{Day of the event:}

1. Arrive at the venue early to ensure technology is working. Log into Tomnod, OSM, and HOT task manager.

2. Once participants arrive, introduce and describe OSM and Tomnod. If responding to a specific crisis, provide information about the event. Present related library materials if appropriate. (10 minutes) 
3. If appropriate, ask participants about their previous experience with OSM or mapping in general. Invite them to share why they've come to the event. (5 minutes)

4. Run through a quick mapping tutorial of OSM and Tomnod. Provide participants with training materials if they prefer to learn at their own speed. (15 minutes)

a. If this is a drop-in style mapathon, you may have to repeat these instructions as new participants arrive.

The remainder of the time is for participants to explore the mapping tools on their own. Float around the room and offer assistance. Encourage working in groups. This is where it is helpful to have a co-facilitator. One facilitator can demonstrate OSM or Tomnod on a presentation screen while the other facilitator individually assists participants.

\section{Promoting a mapathon}

Successful strategies for promoting humanitarian mapathons have included relying on the established community of mappers who have attended introductory workshops and connecting with other groups in the community that have an interest in the affected area. For example, working with the Nepali student group introduced a new audience for library workshops. Nepali students and community members who have a connection to Nepal gathered to map, but also to share stories about Nepal and to support one another. The same was true for community members with a connection to areas devastated by the 2017 Atlantic hurricanes. Participants reported that attending the humanitarian mapathon was helpful to talk to other people from these areas, and to do something useful when they were away from friends and family in crisis. Other participants reported that they liked learning about a place in the world they weren't previously familiar with. If time permits, humanitarian mapathons can be an excellent way to highlight 
library collections by displaying materials related to the affected areas, and by having subject librarians on hand to answer questions. Herman B Wells Library also has plans to partner with the local public library to host mapathons in the public library space and connect with a broader range of participants.

\section{Assessing a mapathon}

At Herman B Wells Library, assessment for mapathons mostly follows the structure for assessment of other library workshops. Participants are asked to sign in at the start of the workshop, indicating their academic rank and department. Following the workshop, participants are requested to complete a brief survey with questions about workshop quality, relevance, skills learned, and a rating of 1-10. All of this information is then logged into Qualtrics. Introductory workshops on OpenStreetMap follow this structure. However, the nature of disaster response mapathons makes this level of assessment data difficult to gather. More often, mapathons are organized very quickly and do not allow time to create surveys. In addition, participants are encouraged to drop in as they please, making post-mapathon surveys difficult to collect, and sign-in sheets easy to ignore. At a minimum, the facilitating librarian will keep a count of the number of participants in attendance and log this number into Qualtrics. In most cases, the librarian will informally ask participants information about their university affiliation, academic rank, and department.

Assessment surveys indicate attendance for humanitarian mapathons has been more academically diverse than other library mapping workshops. Humanitarian mapathons attracted participants from a wide variety of disciplines, as well as university staff, faculty, and community members. In one last-minute mapathon responding to Hurricane Maria, nine 
participants attended, including three graduate students, two undergraduate students, two staff members from the affected area, and two international exchange students. The students and staff came from academic departments in the Humanities, Social Sciences, Area Studies, and Information Technology. This particular mapathon was announced one day before the event took place. Assessment data from workshops at Herman B Wells Library reveals that participants rated humanitarian mapathons an average of 9.4 out of 10 , and would likely recommend mapathons to a friend or colleague.

Contributing to HOT and Tomnod takes very little time to learn, and appeals to a wide variety of participants. Aside from the very real and impactful benefits of contributing to humanitarian mapping campaigns, humanitarian mapathons can be a valuable tool for library outreach and engagement. 


\section{References}

Brown, Greg. 2012. “An Empirical Evaluation of the Spatial Accuracy of Public Participation GIS (PPGIS) Data." Applied Geography 34 (May): 289-94.

Elwood, Sarah, Michael F. Goodchild, and Daniel Z. Sui. 2012. "Researching Volunteered Geographic Information: Spatial Data, Geographic Research, and New Social Practice.” Annals of the Association of American Geographers 102 (3): 571-90. https://doi.org/10.1080/00045608.2011.595657.

Haklay, Mordechai. 2010. "How Good Is Volunteered Geographical Information? A Comparative Study of OpenStreetMap and Ordnance Survey Datasets.” Environment and Planning B Planning and Design, 2010.

Lin, Yu-Wei. 2011. A Qualitative Enquiry into OpenStreetMap Making. New Review of Hypermedia and Multimedia 17 (1): 53-71. https://doi.org/10.1080/13614568.2011.552647.

Mikel. 2010. “Haiti OpenStreetMap Response | OpenStreetMap Blog.” January 14, 2010. https://blog.openstreetmap.org/2010/01/14/haiti-openstreetmap-response/.

“Missing Maps.” n.d. Accessed January 10, 2018. http://www.missingmaps.org/.

National Research Council. 2006. Successful Response Starts with a Map: Improving Geospatial Support for Disaster Management. https://doi.org/10.17226/11793.

Nourbakhsh, Illah, Randy Sargent, Anne Wright, Kathryn Cramer, Brian McClendon, and Michael Jones. 2006. Mapping Disaster Zones. Comments and Opinion. Nature. February 15, 2006. https://doi.org/10.1038/439787a.

“OpenStreetMap.” n.d. OpenStreetMap. Accessed December 22, 2017. http://www.openstreetmap.org/about. 
Peet, Lisa. 2017. Mainland Libraries Aid Maria Victims with Money \& Maps: Crowdsourced Mapping Efforts Help Relief Organizations. Library Journal.

Tulloch, David L. 2007. Many, Many Maps: Empowerment and Online Participatory Mapping. https://doi.org/10.5210/fm.v12i2.1620. 